\title{
THE AQUATIC AND MARSHY FLORA OF THE LOTRU RIVER BASIN (SOUTHERN CARPATHIANS, ROMANIA)
}

\author{
Constantin DR $\breve{A G U L S C U *}$ \\ * "Lucian Blaga" University of Sibiu, Faculty of Sciences, Dr. Ion Raţiu Street 5-7, Sibiu, Sibiu \\ County, Romania, RO-550012, constantindragulescu@yahoo.ro
}

DOI: 10.1515/trser-2015-0015

KEYWORDS: aquatic cormophytes, paludal cormophytes, chorology.

\section{ABSTRACT}

The present paper continues the series of floristic inventories of the main Romanian rivers in the Carpathian Mountains (from which have been published those of Mureș, Sadu, Olt, Someş, Criş, Târnave, Tisa). In this work the author lists 204 plant taxa (hydrophilic, meso-hygrophilic and hygrophilic) identified by the author or by other botanists in the Lotru Valley basin. Noted for each species were life form, floral element, ecological preferences for humidity (U), temperature (T), soil reaction (R), corology and coenology. Bibliographical sources are coded with numbers (see the references at the end of the paper). The sign "!" indicates that the author has seen the plant in that locality.

ZUSAMMENFASSUNG: Die Flora der Gewässer und Sumpfgebiete im Einzugsgebiet des Lotru-Flusses (Südkarpaten, Rumänien).

Mit vorliegender Arbeit wird die floristische Erfassung an den wichtigsten Flüssen im Inneren des Karpatenbogens fortgesetzt, nachdem davor bereits die floristische Erfassung der Flussgebiete des Mureş/Mieresch, Sadu/Zoodt, Olt/Alt, Someş/Somesch, der drei Criş/Kreisch-Flüsse, der beiden Târnava/Kokel-Flüsse und der Tisa/Theiß veröffentlicht wurde. Der Verfasser zählt 204 Taxa hydrophiler, hygrophiler und mesohygrophiler Pflanzen auf, die von ihm oder anderen Botanikern im Einzugsgebiet des LotruFlusses festgestellt wurden. Für jede Art wird Lebensform, floristisches Element, ökologische Ansprüche gegenüber Feuchtigkeit (U), Temperatur (T) und Bodenreaktion sowie Zönologie und Chorologie angegeben. Die Literaturquellen sind mit Hilfe eines Zifferncodes (siehe Literaturverzeichnis am Schluss der Arbeit) vermerkt. Das Ausrufezeichen „!” weist darauf hin, dass der Verfasser die Art an dem betreffenden Ort selbst festgestellt hat. România).

REZUMAT: Flora acvatică şi palustră din bazinul Lotrului (Carpații Meridionali,

Lucrarea continuă seria inventarelor floristice ale principalelor râuri din interiorul arcului carpatic românesc (din care au fost publicate cele ale Mureşului, Sadului, Oltului, Someșelor, Crişurilor, Târnavelor, Tisei). În prezenta lucrare autorul enumeră 204 taxoni vegetali hidrofili, higrofili şi mezo-higrofili identificaţi de el sau de alţi botanişti în bazinul văii Lotrului. La fiecare specie au fost notate bioforma, elementul floristic, preferinţele ecologice faţă de umiditate $(U)$, temperatură $(T)$ şi reacţia solului $(R)$, cenologia şi corologia. Sursele bibliografice sunt codificate cu cifre (a se vedea bibliografia de la sfârşitul lucrării). Semnul ,"! indică faptul că autorul a văzut planta în locul respectiv. 


\section{INTRODUCTION}

This paper continues a series of floristic inventories of the main Romanian rivers in the Carpathian Mountains, like the followings: Mureş (Drăgulescu, 1995a), Sadu (Drăgulescu, 1995b), Criş (Drăgulescu and Macalik, 1997), Olt (Drăgulescu, 1999), Someş (Drăgulescu and Macalik, 1999), Tisa (Drăgulescu et al., 1999), Târnave (Drăgulescu, 2005).

The right side Lotru River tributary of the Olt River has a length of $76.6 \mathrm{~km}$ and a basin of $1,024 \mathrm{~km}^{2}$, and is situated in the core of Meridional Carpathians. Its direction of flow is East-West, separating the crystalline schists of the Lotrului Mountains (to the North) from the Parângului and Căpăţânii mountains (to the South). The climate is mountainous with an average air temperature of $5^{\circ} \mathrm{C}$ and $900 \mathrm{~mm}$ precipitation per year (Ploaie, 1983).

The first data on the Lotru flora were recorded during the second half of the 19th century, provided by the botanists Fuss $(1853,1866)$, Schur (1866), Brândză (1879-1883) and Grecescu (1898). During the 20th century (particularly the second half of this century) the Lotru flora was intensively studied by Ştefureac and colleagues $(1955,1959,1962$, 1969), Pócs (1957, 1961, 1962, 1963, 1967), Buia and colleagues (1943, 1962, 1963), Ciurchea (1962, 1963), Ploaie and colleagues (1983, 1987, 1999, 2004), Drăgulescu (1995) and more recently by Boruz (2004, 2006, 2007, 2008).

The Lotru Basin wetlands comprise the springs, streams, Lotru River, swamps, natural lakes (glacial lakes), dam lakes and ponds. The main areas where these can be found are in the Latoriţa Valley, Voineşiţa Valley, Jidoaia Valley, Mânăileasa Valley, Păscoaia Valley, Repedea Valley, Stan's Valley, Măceşului Valley, Obârşia Lotrului, Latoriţei Lake, Câlcescu Lake, Vidra Lake, Mălaia Lake, Brădişor Lake, Galbenu Lake, Petrimanu Lake, Balindru Lake and Jidoaia Lake.

\section{MATERIALS AND METHODS}

The paper is based on the references (Grecescu, 1909; Fekete and Blattny, 1913; Nyárády, 1955; Oprea, 2005), on herbarium material (Annex 1) and on personal research in the field over the period 1969-2012. The taxonomic data (taxon names, family order) are taken from Săvulescu (1952-1996) with the updates made by Ciocârlan (2000), and the biological (plant bio-forms), phytogeographical (floristic elements), ecological (U, T and R values) and phytocoenological (coenotaxa) data from Sanda et al. (2003). The species chorology is presented in accordance with personal field observations and notes (where the sign "!" is used in the text) or based on the information of other authors (where a figure appears this is the code of the paper listed in the paper references).

\section{RESULTS AND DISCUSSION}

In total, in the Lotru catchment area 204 vegetal taxa were identified that are either hydrophilous, hygrophilous or meso-hygrophylous. These taxa belong to 170 species, eight subspecies, nine varieties, ten forms and seven hybrids. For each species were recorded the bio-form, the floristic element, the ecological preferences concerning humidity (U), temperature (T) and soil $\mathrm{pH}(\mathrm{R})$, coenology and chorology. The bibliographical sources are encoded in figures (see the references). Most of the species are micro-mesothermal and microthermal, euro-ionic and feebly acido-neutrophyle, of Eurasian and circumpolar origin.

Among rare and endangered species are: Thelypteris palustris, Trollius europaeus, Angelica archangelica, Myricaria germanica, Salix aurita, Salix bicolor, Salix daphnoides, Oxycoccus palustris, Empetrum nigrum, Menyanthes trifoliata, Carex brunescens, Carex lepidocarpa, Carex magellanica ssp. irrigua, Carex pauciflora, etc. The species Tozzia alpina ssp. carpatica is included in the Habitats Directive 92/43/CEE concerning conservation of natural habitats and of wild flora and fauna. 


\section{Flora}

\section{Lycopodiaceae}

Lycopodium inundatum L. (Lycopodiella inundata L.) Holub.: Ch, Cp; U5T2, 5R1, Vaccinio-Piceetea, Oxyccoco-Sphagnetea, Scheuchzerio-Caricetea nigrae: Clăbucet Peak (69), Sterpu Peak (69);

\section{Equisetaceae}

Equisetum fluviatile L.: (E. limosum L.): Hh, Cp; U5T3R0, Magnocaricion elatae, Phragmitetea: Obârşia Lotrului (57, !), Mânăileasa Valley (!);

Equisetum hyemale L.: G, Cp; U3.5T2.5R4, Alno-Padion: Latoriţei Gorge (!), Păscoaia Valley (!);

Equisetum palustre L.: G, Cp; U5T2R0, Molinietalia: Groapa Seacă (19), Vidra Lake (!), Obârșia Lotrului 1,430 m (4), Lotru Valley 1,300-1,350 m (4, !), Mânăileasa Valley (!), Voineasa (!); f. fallax Milde: Mânăileasa Valley (!); var. simplicissimum A. Br.: Obârşia Lotrului-Vidra Lake (!), Mânăileasa Valley (!);

Equisetum sylvaticum L.: G, Cp; U3.5T2R0, Alno-Padion, Alnion glutinosae-incanae: Vidra Lake (!), Obârşia Lotrului 1,430 m (4, 57, !), Lotru Valley 1,250-1,350 m (4), Malaia Valley 1,100 m (4);

Equisetum telmateia Ehrh. (E. maximum Lam.): G, Cp; U3.5T2R0, Alno-Padion, Eriophorion latifolii, Filipendulo-Petasition: Brezoi (!), Curmătura Vidruţei (!), Mânăileasa Valley (!);

\section{Athyriaceae}

Matteuccia struthiopteris (L.) Todaro (Struthiopteris filicastrum All.): H, Cp; U4T2R0, Alno-Padion: Brezoi (!), Latoriţei Gorge (!), Ciungetu (!), Coasta lui Rusu 1,850 m (4), Malaia (47, HBZ, !), Săliştea (!), Stan’s Valley (!), Măceşului Valley (!), Mânăileasa Valley (!), Păscoiaia Valley (!), Repedea Valley (!), Voineasa (!);

\section{Aspidiaceae}

Dryopteris cristata (L.) A. Gray: H, Cp; U4T2R3, Scheuchzerio-Caricetalia nigrae: Bucureasa Mare Valley (7), Voineasa (42);

\section{Thelypteridaceae}

Thelypteris palustris Schott (Dryopteris thelypteris (L.) A. Gray): Hh-G, Cp; U4T0R3, Alnion glutinosae, Alno-Padion, Magnocaricion elatae: Vidra, 1,300 m (4, 37);

\section{Ranunculaceae}

Caltha palustris L.: H, Cp; U4.5T0R0, Calthion palustris, Cardamini-Montion, Molinietalia: Câlcescu (2), Coasta lui Rusu 1,700 m (4), Groapa Dengherului (12), Iezeru (2), Mohoru (2), Muntinu Mare (2), Obârşia Lotrului, 1,430 m (4, 57, !), Ştefanu (2), Lotru Valley, 1,220-1,350 m (4), Malaia Valley, 1,000-1,100 m (4, !), Vidra (!), Ştefleşti Peak (!), Voineasa (!), Zănoguţa (2); var. alpina (Schur) Graebn.: Câlcescu Lake (4, 10, 14, 59), Urdele (10);

Ranunculus flammula L.: H, Eua; U4.5T3R0, Agrostion stoloniferae, Caricion canescentinigrae, Magnocaricion elatae: Groapa Seacă to Obârşia Lotrului (19), Lotru Valley, 1,3001,350 m (4);

Ranunculus repens L.: H, Eua; U4T0R0, Agropyro-Rumicion, Alno-Padion, Bidentetalia tripartiti, Calystegion, Molinio-Arrhenatheretea, Phragmitetea, Plantaginetea majoris, Salicetea purpureae: Brezoi (!), Căldarea Coasta lui Rusu 1,850 m (4), Ciobanu, 1,550-1,600 m (4), Ciungetu (!), Corbu (!), Golotreni (!), Groapa Seacă (19), Vidra Lake (!), Lotru (!), 
Malaia (!), Obârşia Lotrului (57, !), Păscoaia (!), Rudaru (Zăvoiul lui Badac) (56), Săliştea (!), Latoriţa Valley (!), Stan’s Valley (!), Măceşului Valley (!), Lotru Valley, 1,250-1,500 m (4, !), Mânăileasa Valley (!), Pravăţu Valley (!), Vasilatu (!), Vidra, 1,250 m (4, 27, !), Voineasa (27, !), Voineşiţa Valley (!), Voineasa-Obârşia Lotrului (57);

Ranunculus sardous Cr.: Th-TH, Eua; U3,5T3R4, Agropyro-Rumicion, Agrostion stoloniferae, Nanocyperion flavescentis: Lotrului Valley, 510 m (7), Muşătoiu (11);

Thalictrum aquilegifolium L.: H, E; U3.5T2.5R4, Adenostylion alliariae, Alno-Padion, Filipendulo-Petasition: Brezoi (!), Gura Lotrului (!), Jidoaia Valley (!), Voineşiţa Valley (!), Vidra (4, 27), Voineasa (27);

Thalictrum lucidum L.: H, Ec; U4.5T3R5, Alnetea glutinosae, Alno-Padion, FilipenduloPetasition, Molinietalia, Salicetea purpureae: Brezoi (44, !); var. heterophyllum (Wimm. and Grab.) Hay.: Brezoi (!); f. peucedanifolium (Gris. and Sch.) A. Nyar.: Golotreni (!);

Trollius europaeus L. ssp. europaeus: H, E; U4T2R4, Calthion palustris, Molinietalia: Balţuri (6), Dobrunu (6), Găurile (10), Mohorul (10), Voineasa (!), Zănoguţa (10);

\section{Betulaceae}

Alnus glutinosa (L.) Gaerter: Mph-mPh, Eua; U5T3R3, Alnion glutinosae, Alno-Padion: Brezoi (!), Ciungetu (!), Corbu (!), Gura Lotrului (!), Lotrului Valley, 510 m (7), Malaia (!), Păscoaia (!), Săliştea (!), Stan’s Valley (!), Măceşului Valley (!), Bucureasa Valley (7), Malaia Valley (7, !), Malaia Valley $667 \mathrm{~m}$ (4);

Alnus incana (L.) Moench: Mph-mPh, E; U4T2R4, Alno-Padion, Salicion albae: Latoriţei Gorge (!), Ciungetu (!), Malaia (!), Săliştea (!), Voineasa (6, !), Voineasa-Obârşia Lotrului (57, !), Bucureasa Valley (7), Lotrului Valley, 510-1,350 m (4, 7), Malaia Valley, 764 m (4), Voineşiţa Valley, 1,100 m (!);

\section{Caryophyllaceae}

Lychnis flos-cuculi L.: H, Eua; U4T2.5R0, Magnocaricion elatae, Molinietalia, MolinioArrhenatheretea: Brezoi (!), Golotreni on Narăţu Mountain (30), Gura Lotrului (!), Obârşia Lotrului (!), Lotrului Valley, 1,350 m (4), Malaia Valley, 1,100 m (4, !), Voineasa (!);

Myosoton aquaticum (L.) Moench (Stellaria aquatica (L.) Scop.): H, Eua; U4T3R0, AlnoPadion, Bidention tripartiti, Salicion albae, Senecion fluviatilis: Ciungetu (!), Malaia (!), Robu Mountain (55), Rudaru (Zăvoiul lui Badac) (56), Măceşului Valley (!), Deluşelu Valley (!), Jidoaia Valley (!), Malaia Valley (7, !), Mănăileasa Valley (!), Rânjeu Mare Valley (!), Rânjeu Mic Valley (!), Voineşiţa Valley (!), Balindru Peak (!), Buceciu Peak (!), Clăbucet Peak (!), Conţu Mare Peak (!), Cristeşti Peak (!), Negovanu Mare Peak (!), Ştefleşti Peak (!), Voineagu Cătănesii Peak (!), Voineşiţa Peak (!), Voineasa (!);

Spergularia rubra (L.) J. and C. Presl.: Th-H, Cp; U4T3R4, Bidentetea tripartiti, Nanocyperion flavescentis, Plantaginetea majoris: Brezoi (!), Obârşia Lotrului (57), Văleanu (12);

Stellaria uliginosa Murray (S. alsine Grimm.): H, Cp; U4.5T2.5R2.5, Cardamini-Montion: tail of Vidra Lake (!), Lotrului Valley, 1,230-1,350 m alt. (4, 37), Rânjeu Mare Valley (!), Voineşiţa Valley (!), Voineasa (!);

\section{Polygonaceae}

Polygonum bistorta L.: G, Eua; U4T2.5R3, Calthion palustris, Molinietalia, TrisetoPolygonion: Urdele-Cărbunele (10), Vidra (4, 27), Negovanu Mare Peak (68, !), Sterpu Peak (73, 74, HF), Ştefleşti Peak (68, HDRG, !), Voineasa (27, !); f puberulum Beck: Negovanu Mare Peak (68, !)

Polygonum hydropiper L.: Th, Cp; U4.5T3R4, Alnetea glutinosae, Bidention tripartiti, Salicion albae: Brezoi (!), Ciungetu (!), Mălaia (!), Păscoaia (!), Săliştea (!), Stan’s Valley 
(!), Măceşului Valley (!), Latoriţa Valley (!), Lotrului Valley, 510 m (7), Mânăileasa Valley (!), Voineasa (!), Voineşiţa (!);

Polygonum lapathifolium L. ssp. lapathifolium: Th, Cosm; U4T0R3, Bidention tripartiti, Polygono-Chenopodion polyspermi, Sisymbrion officinalis; Brezoi (!), Malaia (!), Măceşului Valley (!);

Polygonum mite Schrank: Th, E; U5T3R4, Bidentetalia tripartiti; Brezoi (!);

Polygonum persicaria L.: Th, Eua; U4.5T3R0, Phragmitetea, Polygono-Chenopodietalia, Salicetalia purpureae: Brezoi (!), Mălaia (!);

Rumex conglomeratus Murray: H, Cp; U4T3R4, Agropyro-Rumicion, Bidention tripartiti; Brezoi (!), Ciungetu (!), Corbu (!), Malaia (!), Săliştea (!), Stan’s Valley (!), Măceşului Valley (!), Mânăileasa Valley (!), Voineasa (!);

Rumex crispus L.: H, Eua; U4T3R0, Agropyro-Rumicion, Arrhenatherion elatioris: Brezoi (!), Corbu (!), Golotreni (!), Gura Lotrului (!), Măceşului Valley (!), Mânăileasa Valley (!), Voineasa (!), Voineşiţa (!);

Rumex maritimus L.: Th, Cosm; U5T3.5R4.5, Bidentetea tripartiti, Plantaginetea majoris, Brezoi (!);

Rumex sanguineus L.: H, E; U4T3R4, Alno-Padion, Fagetalia silvaticae: Brezoi (!), Golotreni (!), Mânăileasa Valley (!), Voineasa (!); f. atropurpureus Aschers. Mânăileasa Valley (!), Voineasa (!);

\section{Saxifragaceae}

Chrysosplenium alpinum Schur (C. oppositifolium L. ssp. alpinum (Schur) Jav.: H, Carp; U4T3R3.5, Androsacion alpinae, Montio-Cardaminetalia: Circul Câlcescu (14);

Chrysosplenium alternifolium L.: H, Cp; U4T2R4, Alno-Padion, Fagetalia silvaticae: Brezoi (!), Coasta Bengăi (10), Mohorul (10), Obârşia Lotrului 1,350 m (4, !), Găuri Valley (4), Vidra 1,200 m (4, !), Voineasa (!), Ştefleşti Peak (68, HDRG, HRDG, !);

Saxifraga stellaris L. ssp. robusta (Engler) Gremli: Ch, Eua (arct-alp); U5T1.5R3, Cardamini-Montion; Circul Câlcescu (14), Coasta Bengăi (10), Dobrun (HBZ), Groapa Seacă-Obârşia Lotrului (19), Obârşia Lotrului 1,350 m (4, !), Păpuşa, 1,800-2,000 m (4), Piatra Tăiată, 2,250 m (4), Puru (10), Setea Mare 2,340 m (4), Găuri Valley (4), Vidra, 1,220-1,300 m (4, 27, !), Rânjeu Mare Valley (!), Buceciu Peak (!), Clăbucet Peak (68, !), Conţu Mare Peak (68, HDRG, HBZ, HF, !), Negovanu Mare Peak (68, !), Ştefleşti Peak (68, HRDG, !), Voineagu Cătănesii (68, !);

\section{Parnassiaceae}

Parnassia palustris L.: H, Cp; U4.5T2R4.5, Caricetalia davallianae, Molinion coeruleae, Tofieldietalia: Boarneşu (10), Latoriţei Gorge (!), Câlcescu (2), Coasta lui Rusu, 2,150 m (4), Coasta Petresii, 1,800 m (2), Coasta Pietroasă (2), Iezeru (2), Mogoşu (2, 10, 86), Mohoru (2), Obârşia Lotrului (4, !), Pietrele (2), Poliţi, 2,240 m (4), Ştefanu (2), Urdele (10), Găuri Valley, 2,125 m (4), Lotrului Valley, 1,300 m (4), Zănoguţa (2);

\section{Rosaceae}

Filipendula ulmaria (L.) Maxim.: H, Eua; U4.5T2R0, Alno-Padion, Filipendulo-Petasition, Molinietalia: Malaia (!), Muşătoiul (10), Obârşia Lotrului (57, !), Lotrului Valley, 1,3001,350 m (4), Piatra Albă-Tărtărău Peak (62); ssp. denudata (J. and C. Presl.) Hayek.: Mirăuţu Mountain (!), Vidra-Obârşia Lotrului (!), Miru Valley (!), Mânăileasa Valley (!), Puru Valley (!), Voineasa (!);

Geum rivale L.: H, Cp; U4.5T0R4.5, Adenostylion alliariae, Calthion palustris, FilipenduloPetasition; Ciobanu, 1,790-1,850 m (4), Coasta lui Rusu, 1,700 m (4), Căldarea Coasta lui 
Rusu 1,850 m (4), Târnovu Mountain (HBZ), Obârşia Lotrului (4, !), Păpuşa 1,800 m (4), Lotrului Valley, 1,300-1,500 m (4, !), Vidra, 1,250 m (4, 27, !), Negovanu Mare Peak (68, HDRG, !), Ştefleşti Peak (68, !), Voineagu Cătănesii (68, HDRG, !), Voineasa (27, 59);

Potentilla anserina L.: H, Cosm; U4T3R4, Bidentetalia tripartiti, Molinietalia, Nanocyperetalia, Plataginetalia majoris: Brezoi (!), Gura Lotrului (!), Stan’s Valley (!), Lotrului Valley, 1,300 m (4), Voineasa (!), Voineşiţa (!);

Potentilla supina L.: TH-H, Eua-sM; U4T3R0, Bidention tripartiti, Nanocyperion flavescentis; Gara Lotru (13);

Fabaceae

Trifolium hybridum L. ssp. hybridum: H, Atl-E; U3.5T3R4, Agropyro-Rumicion, Agrostion stoloniferae, Calthion palustris: Brădişor (!), Brezoi (!), Golotreni (59), Muşătoiu (10), Mânăileasa Valley (!), Voineşiţa Valley (!), Văleanu (12), Voineasa (!);

\section{Lythraceae}

Lythrum portula (L.) D. A. Webb. (Peplis portula L.): Th, Atl-M; U4T3R0, Nanocyperion flavescentis; Gura Lotrului (!);

Lythrum salicaria L.: H, Cp; U4T3R0, Alnetea glutinosae, Filipendulo-Petasition, Molinietalia, Phragmitetea, Salicetea purpureae: Brezoi (!), Ciungetu (!), Gura Lotrului (!), Malaia (!), Golotreni (!), Păscoaia (!), Săliştea (!), Stan’s Valley (!), Măceşului Valley (!), Lotrului Valley, 1,350 m (4), Voineasa (!), Voineşiţa (!); var. tomentosum D. C.: Golotreni (30);

Onagraceae

Epilobium alsinifolium Vill.: H, E (arct-alp); U5T1.5R0, Montio-Cardaminetea, Căldarea Coasta lui Rusu, 1,850 m (4), Ciobanu, 1,850 m (4), Obârşia Lotrului, 1,350 m (4), Găuri Valley (10, 27), Clăbucet Peak (68, HDRG, !);

Epilobium anagalidifolium Lam. (E. alpinum auct. non L.): H, Cp (arct-alp); U4T1.5R0, Androsacetalia alpinae, Cardamini-Montion: Obârşia Lotrului (!);

Epilobium ciliatum Rafin (E. adenocaulon Hausskn, E. hirsutum var. adenocaulon Hausskn.): H, Adv; U4T3R3, Filipendulo-Petasition, Molinietalia, Phragmitetea: Brezoi (!);

Epilobium hirsutum L.: H, Eua; U4T3R3, Filipendulo-Petasition, Phragmitetea: Ciungetu (!);

Epilobium nutans F. W. Schmidt: H, E (alp); U5T2R2, Cardamini-Montion, Sphagnion fusci; Groapa Seacă-Obârşia Lotrului (19), Obârşia Lotrului (!), Găuri Valley (4), Vidra (4), Ştefleşti Peak $(68, !)$, Voineagu Cătănesii $(68, !)$;

Epilobium obscurum Schreber: H, E; U5T0R2, Cardamini-Montion, Epilobietea angustifolii, Glycerio-Sparganion: Brezoi (!), Ciungetu (!), Malaia (!), Măceşului Valley (!), Mânăileasa Valley (!), Voineasa (!), Voineşiţa (!);

Epilobium palustre L.: H, Cp; U5T0R2, Calthion palustris, Magnocaricion elatae, Scheuchzerio-Caricetalia nigrae: Lotrului Valley, 1,250-1,300 m (4), Malaia Valley, 1,000 m (4, !), Mânăileasa Valley (!);

Epilobium parviflorum Schreber: H, Eua; U5T3R4.5, Glycerio-Sparganion, Phragmitetea: Lotrului Valley, 1,300-1,350 m (4), Mănăilesa Valley (!);

Epilobium roseum Schreber: H, Eua; U4.5T3R4.5, Glycerio-Sparganion: Câlcescu Lake, 1,930 m (2), Iezeru, 1,935 m (2), Muntinu Mic, 1,910 m (2), Ştefanu, 1,910 m (2);

Geraniaceae

Geranium palustre Torn.: H, Eua-C; U4T3R4.5, Filipendulo-Petasition: Rânjeu Mare Valley, 1,300 m (!), Voineşiţa Valley (!); 


\section{Apiaceae}

Angelica archangelica L.: TH-H, Eua (bor); U4.5T2.5R0, Adenostyletalia, FilipenduloPetasition: Ciobanu, 1,400-1,500 m (4), Câlcescu (10), Latoriței Gorge (!), Coasta lui Rusu, 1,700 m (4), Dobrun (!), Jidu (!), Obârşia Lotrului (!), Măceşului Valley (!), Jidoaia Valley (!), Voineşiţa Valley, Vidra, 1,250 m (4);

Angelica sylvestris L. ssp. sylvestris: TH-H, Eua; U4T3R3, Alno-Padion, Molinietalia: Groapa Seacă-Obârşia Lotrului (19), Galbenu Stream (10), Stan’s Valley (!), Măceşului Valley (!); ssp. montana (Brot.) Archang.: Coasta lui Rusu, 1,700 m (4), Vidra, 1,250 m (4);

Angelica x mixta Nyar. (archangelica x sylvestris): LakeVidra-Obârşia Lotrului (!);

Oenanthe peucedanifolia Pollich (O. stenoloba Schur): H, D-B; U4T0R4.5, Molinietalia: Lotrului Valley, 1,400 m alt. (4, 37);

\section{Hypericaceae}

Hypericum tetrapterum Fries. (H. quadrangulum L.): H, E; U4T3R4, FilipenduloPetasition, Glycerio-Sparganion, Magnocaricion elatae: Brezoi (!), Ciobanu, 1,700-1,850 m (4), Narăţu Mountain (30);

\section{Tamaricaceae}

Myricaria germanica (L.) Desv.: nPh, Eua; U0T0R4.5, Salicion eleagni: Obârşia Lotrului (57, !), Latoriţei Valley (!) under Târnovu Mountain (HBZ), Lotrului Valley, 1,300 m (4, !), Mânăileasa Valley (!), Voineşiţa Valley (!), Voineasa (!);

\section{Brassicaceae}

Cardamine amara L. ssp. amara: H, Eua; U5T0R0, Alno-Padion, Cardamini-Montion: Circul Câlcescu (86), Obârşia Lotrului, 1,350-1,430 m (4), Găuri Valley (4), Haneşu Valley (!), Lotrului Valley, 1,200-1,300 m (4, !), Vidra (!), Cristeşti Peak (!), Ştefleşti Peak (68, !); ssp. opicii (J. and C. Presl.) Celak.: H, Ec; Câlcescu, 1,930 m (2), Coasta lui Rusu 1,700 m (4), Iezeru, 1,935 m (2), Muntinu Mic, 1,910 m (2), Ştefanu, 1,910 m (2), Găuri Valley (4), Lotrului Valley, 1,300-1,350 m (4),

Cardamine pratensis L. ssp. pratensis: H, Cp; U5T3R0, Molinio-Arrhenatheretea: Câlcescu, 1,930 m (2), Coasta Bengăi (10), Găuri (4, 10, 27), Muntinu Mare (10), Muntinu Mic (10), Ştefanu, 1,910 m (2); ssp. matthioli (Moretti) Nyman (ssp. hayneana (Welw.) D. E. Schultz): H, E; Clăbucet Peak (68, !), Negovanu Mare Peak (!); ssp. rivularis (Schur) Nyman: H, Carp-B: Circul Câlcescu (14), Dobrun (HBZ), Păpuşa, 1,800-1,850 m (4), Găuri Valley, 2,060 m alt. (4, 37), Lotrului Valley, 1,300-1,400 m (4, 37);

Rorippa austriaca (Crantz) Besser: H, P; U4T3.5R4, Agropyro-Rumicion, Bidentetea tripartiti, Plantaginetea majoris, Senecion fluviatilis; Brezoi (!);

Rorippa sylvestris (L.) Besser ssp. sylvestris: H, Eua; U4T3R4, Agropyro-Rumicion: Brădişor (!), Brezoi (!), Ciungetu (!), Golotreni (!), Groapa Seacă-Obârşia Lotrului (19), Malaia (!), Obârşia Lotrului (57, !), Păscoaia (!), Săliştea (!), Măceşului Valley (!), Lotrului Valley 510-1,300 m (4, 7, !), Mânăileasa Valley (!), Rânjeu Mare Valley (!), Rânjeu Mic Valley (!), Voineşiţa Valley (!), Vasilatu (!), Voineasa (!), Voineşiţa (!); ssp. kerneri (Menyh.) Soo: H, Pn: Lotrului Valley, 510 m (7);

\section{Salicaceae}

Populus alba L.: Mph-mPh, Eua; U3.5T3R3, Salicetalia purpureae: Brădişor (!), Golotreni (!), Gura Lotrului (!), Voineşiţa Valley (!);

Salix alba L. ssp. alba: Mph-mPh, Eua; U5T3R4, Alno-Padion, Salicion albae: Brezoi (!), Ciungetu (!), Corbu (!), Golotreni (!), Lotru (!), Malaia (!), Măceşului Valley (!), 
Mânăileasa Valley (!), Voineşiţa Valley (!), Voineasa (!);

Salix aurita L.: mPh, E; U4.5T0R2, Alnetea glutinosae: Lotrului Valley, 1,300 m alt. $(4,37)$;

Salix bicolor Willd. (S. phylicifolia auct.): mPh, Eua (bor); U4T1.5R2, Vaccinio-Piceetea: Câlcescu Lake (1, 4, 6, 8, 10, 14);

Salix cinerea L.: mPh, Eua; U5T3R3, Alnetea glutinosae, Alno-Padion; Pravăţu Mare (1), Sărăcinu Mic (1), Vidra (52, !); f. aquatica (Sm.) Koch: Lotrului Valley (1);

Salix daphnoides Vill.: mPh, Eua; U4.5T2.5R4.5, Salicion eleagni: Pasul Turnu-Roşu (90, 74);

Salix eleagnos Scop. (S. incana Schrank): mPh, Ec; U4T3R4.5, Salicion eleagni; Brădişor (!), Gura Lotrului (!), Malaia (!), Latoriţa Valley (!), Lotrului Valley (4, 59), Voineşiţa Valley (!);

Salix fragilis L.: mPh-MPh, Eua; U4.5T3R4, Alno-Padion, Salicion albae, Salicion triandrae: Brezoi (!), Golotreni (!), Malaia (!), Măceşului Valley (!), Mânăileasa Valley (!), Voineasa (!), Voineşiţa (!); f. discolor Kern.: Măceşului Valley (!);

Salix purpurea L. ssp. purpurea: $\mathrm{mPh}$, Eua; U5T3R4.5, Salicetalia purpureae; Brădişor (!), Brezoi (!), Latoriţei Gorge (!), Ciungetu (!), Corbu (!), Golotreni (!), Gura Lotrului (!), Malaia (!), Obârşia Lotrului (!), Măceşului Valley (!), Latoriţa Valley (!), Lotrului Valley, 1,300 m (4), Mânăileasa Valley (!), Voineşiţa Valley (!), Vidra (1), Voineasa (!), Voineşiţa (!);

Salix silesiaca Willd.: mPh, Carp-B-Sud; U4T2R2, Adenostyletalia, Pinion mugi; Ciobanu, 1,500 m (4), Groapa Seacă-Obârşia Lotrului (19), Pravăţu Mare (1), Sărăcinu Mare (1), Sărăcinu Mic (1), Lotrului Valley, 1,300-1,450 m (4), Mânăileasa Valley (!),Vidra (1, 27), Sterpu Peak (!), Voineasa (27);

Salix starkeana Willd.: nPh (Ch), Eua (bor); U4T2.5R2, Oxycocco-Sphagnetea; Sărăcinu Mare (1), Sărăcinu Mic (1);

Salix triandra L. emend. Ser. ssp. triandra: mPh, Eua; U5T3R0, Salicion triandrae: Malaia (!), Păscoaia (!), Săliştea (!), Stan’s Valley (!), Lotrului Valley, 1,300 m (4), Vidra (1); ssp. amygdalina (L.) Schubl. and G. Martens (ssp. discolor (Koch) Arcangeli): Măceşului Valley (!);

Salix viminalis L.: mPh, Eua; U5T2.5R4.5, Salicion triandrae; Brezoi (!), Ciungetu (!), Gura Lotrului (!);

Salix x capreola Kern. (aurita x caprea): Vidra (1);

Salix $x$ multinervis Doll. (aurita $x$ cinerea): Vidra (1);

Salix $x$ reichardtii Kern. (caprea $x$ cinerea): Vidra (1);

Salix $x$ rubens Schrank. (alba x fragilis): Mălaia (!), Voineşiţa (!);

Salix x subcaprea Anderss. (caprea x silesiaca): Lacul Vidra Lake (!), Obârşia Lotrului (!), Sărăcinu Mare (1), Sărăcinu Mic (1), Mânăileasa Valley (!), Vidra (1);

Salix x subcinerea Anderss. (cinerea x silesiaca): Vidra (1);

Ericaceae

Oxycoccus palustris Pers. (Vaccinium oxycoccos L. ssp. oxycoccos): Ch, Cp; U5T0R2, Oxycocco-Sphagnetea: Iezerul Latoriţei (8);

Empetraceae

Empetrum nigrum L.: Ch-nPh, Cp; U4T0R0, Junipero-Bruckenthalion; Circul Câlcescu (2, 8, 10, 14), Iezeru (10, 14), Mohoru (14), Obârşia Lotrului-Vidra (52), Petrimanu, 1,850 m (2), Puru, 1,850 m (2, 14); 


\section{Primulaceae}

Lysimachia nummularia L.: Ch, Eua; U4T3R0, Alnetea glutinosae, Alno-Padion, Bidentetea tripartiti, Calthion palustris, Filipendulo-Petasition, Molinietalia, Phragmitetea, Plantaginetea majoris, Querco-Fagetea, Salicion albae: Brezoi (!), Latoriţei Gorge (!), Ciungetu (!), Golotreni (30, !), Gura Lotrului (!), Malaia (!), Păscoaia (!), Stan’s Valley (!), Măceşului Valley (!), Lotrului Valley 510-1,350 m (4, 7, !);

Lysimachia vulgaris L.: H(-Hh), Eua; U5T0R0, Alnetea glutinosae, Molinietalia, Phragmitetea, Salicetea purpureae, Scheuchzerio-Caricetea nigrae; Brezoi (!), Golotreni (30, !), Stan’s Valley (!), Măceşului Valley (!), Voineasa (!);

\section{Gentianaceae}

Centaurium pulchellum (Swartz) Druce: Th-TH, Eua; U4T3.5R4, Isoeto-Nanojuncetea; Ciungetu (!);

\section{Solanaceae}

Solanum dulcamara L.: Ch $(\mathrm{nPh})$, Eua; U4.5T3R4, Alnetea glutinosae, Alno-Padion, Bidentetea tripartite, Calystegion, Epilobietalia angustifolii, Phragmition australis: Brezoi (!), Golotreni (!), Mălaia (!), Păscoaia (!), Săliştea (!), Voineşiţa Valley (!);

\section{Convolvulaceae}

Calystegia sepium (L.) R.Br.: G(H), Eua; U4.5T3R4, Calystegion, Salicion albae, Arction lappae: Brezoi (!), Ciungetu (!), Golotreni (!), Gura Lotrului (!), Malaia (!);

\section{Menyanthaceae}

Menyanthes trifoliata L.: Hh, Cp; U5T0R0, Magnocaricion elatae, ScheuchzerioCaricetalia nigrae: Violeta Lake in Latoriţa Valley $(8,25)$;

\section{Boraginaceae}

Myosotis caespitosa K. F. Schultz: Th-TH, Cp; U4.5T0R0, Magnocaricion, Phragmition; Săliştea (!);

Myosotis nemorosa Besser (M. palustris var. nemorosa (Bess.) Schmalh.): TH-H, Eua; U5T3R0, Calthion; Negovanu Mare Peak (68, HDRG, !);

Myosotis scorpioides L. (M. palustris (L.) Hill): H(Hh), Eua; U5T3R0, Alnetea glutinosae, Calthion palustris, Molinietalia, Phragmitetea: Brezoi (!), Ciungetu (!), Golotreni (!), Groapa Seacă-Obârşia Lotrului (19), Gura Lotrului (!), Vidra Lake (!), Malaia (!), Obârşia Lotrului (4, !), Găuri Valley (4), Latoriţa Valley (!), Lotrului Valley, 1,250-1,350 m (4, !), Malaia Valley, 1,100 m (4, !), Mânăileasa Valley (!), Ştefleşti Peak (68, HDRG, !);

Symphytum officinale L. ssp. officinale: H, Eua; U4T3R0, Molinietalia, Phragmitetea: Golotreni (!);

\section{Lamiaceae}

Lycopus europaeus L.: H (Hh), Eua; U5T3R0, Bidentetea tripartiti, Phragmitetea, Salicetea purpureae: Ciungetu (!), Golotreni (30, !), Malaia (!), Săliştea (!), Măceşului Valley (!), Mânăileasa Valley (!), Voineasa (!);

Mentha aquatica L.: H (Hh), E; U5T3R0, Alnetea glutinosae, Molinietalia, Phragmitetea, Salicion albae: Lotru (!), Golotreni (!); var. riparia Schreb.: Brezoi (!), Săliştea (!);

Mentha arvensis L. ssp. arvensis (ssp. agrestis (Sole) Briq.): H(G), Cp; U4T3R0, Calthion palustris, Molinietalia, Phragmitetea, Secalietea: Golotreni (30, !), Malaia (!), Măceşului Valley (!), Voineasa (!); 
Mentha longifolia (L.) Hudson ssp. longifolia: H(G), Eua; U4.5T3R0, Agropyro-Rumicion, Bidentetea tripartiti, Chenopodietea, Filipendulo-Petasition, Glycerio-Sparganion, Molinietalia: Brezoi (!), Ciobanu, 1,600 m (4), Ciungetu (!), Gura Lotrului (!), Malaia (!), Păscoaia (!), Săliştea (!), Stan’s Valley (!), Măceşului Valley (!), Latoriţa Valley (!), Lotrului Valley, 510-1,300 m (4, 7, !), Mânăileasa Valley (!), Voineşiţa Valley (!), Voineasa (!); var. horridula Briq.: Măceşului Valley (!);

Mentha pulegium L.: H, Eua (sM); U4T3R5, Isoeto-Nanojuncetea, Nanocyperion flavescentis; Brezoi (!), Golotreni (!); var. hirsutum (Per.) Lab.: Golotreni (!);

Scutellaria galericulata L.: H, Cp; U4T3R4, Magnocaricion elatae, Molinietalia, Phragmitetea Brezoi (30, 59, !);

Callitrichaceae

Callitriche cophocarpa Sendtner (C. polymorpha Lonnr.): Th-H (Hh), Eua; U6T3R0, Nanocyperion flavescentis, Potamion: Malaia (!);

Callitriche palustris L. (C. verna L.): Th-H (Hh), Cp; U6T3R0, Nanocyperion flavescentis, Potamion: Câlcescu (2), Iezeru (2, 10);

Scrophulariaceae

Gratiola officinalis L.: H, Cp; U4.5T3R4, Magnocaricion elatae, Molinion coeruleae, Nanocyperetalia, Phragmitetea: Gura Lotrului (!);

Tozzia alpina L. ssp. carpatica (Woloszczak) Hayek: H, Carp-B; U4T2R4.5, Adenostyletalia, Cardamini-Montion; Vidra, 1,250 m (4), Voineagu Cătănesii (68, $\underline{\text { HDRG}}$ !), Voineasa (!);

Veronica anagallis-aquatica L.: H (Hh), Cp; U5T0R4, Bidentetea tripartiti, GlycerioSparganion, Phragmitetea: Gura Lotrului (!), Săliştea (!), Mânăileasa Valley (!), Voineasa (!);

Veronica beccabunga L.: H (Hh), Eua; U5T3R4, Bidentetea tripartiti, Glycerio-Sparganion, Salicetalia purpureae: Brezoi (!), Ciungetu (!), Dengheru-Păpuşa, 1,910 m (2), Groapa Dengherului (12), Gura Lotrului (!), Săliştea (!), Măceşului Valley (!), Găuri Valley (4), Mânăileasa Valley (!), Voineşiţa Valley (!), Voineasa (!);

\section{Lentibulariaceae}

Pinguicula alpina L.: H, Eua (arct-alp); U4T0R4, Caricion davallianae, Cratoneurion commutati, Eriophorion latifolii, Seslerietalia coeruleae: Lotrului Valley, 1,300 m alt. (4, 37);

Pinguicula vulgaris L.: H, Cp; U3,5T0R4, Caricion canescenti-nigrae, Caricion davallianae, Cratoneurion commutati, Tofieldietalia; Groapa Dengherului (12);

Rubiaceae

Galium palustre L. ssp. palustre: H, Cp; U5T3R0, Magnocaricion elatae, Molinietalia: Brezoi (!), Gura Lotrului (!), Obârşia Lotrului (!), Gura Pravăţului (4, 27, !), Lotrului Valley, 1,250 m (4), Malaia Valley, 1,100 m (4, !), Mânăileasa Valley (!);

Galium uliginosum L.: H, Eua; U4.5T3R4, Calthion palustris, Magnocaricion elatae, Molinietalia, Scheuchzerio-Caricetalia nigrae: Găuri Valley (4), Lotrului Valley, 1,2501,350 m (4, !), Malaia Valley, 1,100 m (4, !);

Valerianaceae

Valeriana officinalis L.: H, Eua (sM); U4T3R4, Alnetea glutinosae, Alno-Padion, Filipendulo-Petasition, Magnocaricion elatae, Molinietalia: Latoriţei Gorge (!), Coasta lui Rusu at Poliţi (36), Găuri Valley, 2,080-2,165 m (4, 37), Obârşia Lotrului (!), Păscoaia (!), Săliştea (!), Stan’s Valley (!), Jidoaia Valley (!), Voineşiţa Valley (!); var. latifolia Vahl: f. altissima (Hornem.) Koch: Brezoi (30, 59); 
Valeriana sambucifolia Mikan fil. (V. officinalis L. ssp. sambucifolia (Mikan fil.) Celak.): H, Ec; U4T2R3.5, Adenostyletalia, Fagion, Filipendulo-Petasition: Latoriţei Gorge (!), Căldarea Coasta lui Rusu, 1,850 m (4), Coasta lui Rusu, 1,700 m (4), Ciobanu, 1,850 m (4), Malaia (!), Măceşului Valley (!), Mânăileasa Valley (!), Vidra, 1,250 m (4), Voineasa (!), Jidoaia Valley $(27,59)$;

\section{Asteraceae}

Bidens tripartita L.: Th, Eua; U4.5T3R0, Bidentetea tripartiti, Chenopodio-Scleranthetea, Nanocyperion flavescentis: Brezoi (!), Ciungetu (!), Gura Lotrului (!), Malaia (!), Lotrului Valley, 510 m (7), Stan’s Valley (!), Măceşului Valley (!), Voineasa (!), Voineşiţa (!); f. pumila (Roth) Nyar.: Mânăileasa Valley upstream of Voineasa (!);

Bidens vulgata E. L. Greene: Th, Adv; U5T0R0, Bidention; col: Gura Lotrului (!);

Carduus personatus (L.) Jacq. ssp. personatus: H, Ec; U4.5T2.5R4.5, Adenostylion alliariae, Alno-Padion, Filipendulo-Petasition; Ciungetu (!), Curmătura Vidruţei (!), Vidra Lake (!), Malaia (!), Păscoaia (!), Săliştea (!), Măceşului Valley (!), Mânăileasa Valley (!), Voineasa (!);

Cirsium canum (L.) All.: G, Eua-C; U4.5T3R4.5, Alno-Padion, Magnocaricion elatae, Molinietalia: Brezoi (!), Săliştea (!);

Cirsium erisithales (Jacq.) Scop.: H, Ec (mont); U3,5T3R4.5, Fagetalia silvaticae, Filipendulo-Petasition; Brezoi (!), Latoriţei Gorge (!), Ciungetu (HBZ, !), Golotreni (47, !), Malaia (!), Săliştea (!), Deluşelu Valley (!), Măceşului Valley (!), Mânăileasa Valley (!), Rânjeu Mare Valley (!), Rânjeu Mic Valley (!), Voineşiţa Valley (!), Vidra (!), Voineasa (!), Jidoaia Valley (27, !);

Cirsium oleraceum (L.) Scop.: H, Eua; U4T3R4, Alno-Padion, Calthion palustris, Filipendulo-Petasition, Molinietalia: Brezoi (!), Voineasa (42);

Cirsium palustre (L.) Scop.: TH, Eua; U4.5T3R2.5, Alnetea glutinosae, Epilobietalia angustifolii, Molinietalia, Phragmitetea: Curmătura Vidruţei (!), Groapa Seacă-Obârşia Lotrului (19), Vidra Lake (!), Obârşia Lotrului-Vidra Lake (!), Galbenu Stream (10), Mânăileasa Valley (!), Pravăţu Valley (!), Rânjeu Mare Valley (!), Voineşiţa Valley (!), Voineasa (!);

Cirsium rivulare (Jacq.) Link.: H, Ec (mont); U4T3.5R0, Alnetea glutinosae, Calthion palustris, Magnocaricion elatae, Molinietalia: Săliştea (!);

Cirsium waldsteinii Rouy: H, Alp-Carp; U4T2R2, Adenostylion alliariae, Caricion curvulae, Filipendulo-Petasition; Coasta Bengăi (2, 86), Cracul Butirii-Malaia (55), Gaura Mohorului (10), Micaia (2), Mohoru (2), Păpuşa (10), Părăginosu (2), Galbenu Stream (10), Voineşiţa Valley (!), Voineasa (59, !), Jidoaia Valley (27, 59, !);

Crepis paludosa (L.) Moench: H, E (mont); U4.5T0R4.5, Adenostyletalia, Alnetea glutinosae, Alno-Padion, Calthion palustris, Montio-Cardaminetea; Căldarea Mohorului (2), Mohoru (10), Păpuşa (10), Lotrului Valley, 1,300 m alt. (37);

Eupatorium cannabinum L.: H, Eua; U4T3R0, Alnion glutinosae, Epilobietea angustifolii, Filipendulo-Petasition, Phragmitetea, Salicetalia purpureae: Brădişor (!), Brezoi (31, !), Ciungetu (!), Lotru (!), Malaia (!), Păscoaia (!), Săliştea (!), Stan’s Valley (!), Măceşului Valley (!), Latoriţa Valley (!), Mânăileasa Valley (!), Voineasa (!);

Gnaphalium uliginosum L.: Th, Eua; U5T3R4, Nanocyperetalia: Bora (2), Brezoi (31), Coasta Pietroasă (2), Dengheru (2), Gaura Mohorului-Urdele, 2,120 m (2), Micaia (2), Mirăuţu (2), Mogoşu-Turcinu Mare, 1,920 m (2), Mohoru (2, 11), Muntinu Mic (2), Muşătoiu (2, 86), Nopteasa (11), Păpuşa (2, 86), Puru (2, 86), Zănoguţa (2); 
Petasites albus (L.) Gaertner: G, Eua; U3.5T0R0, Alno-Padion, Fagion: Golotreni (47), Păscoaia (!), Jidoaia Valley (!), Latoriţei Valley (!), Repedea Valley (HBZ, !), Voineşiţa Valley (!), Zmeurătu (!);

Petasites hybridus (L.) P. Gaertner, B. Meyer and Scherb.: G, Eua; U5T3R3, Adenostyletalia, Alno-Padion, Filipendulo-Petasition: Brezoi (!), Ciungetu (!), Malaia (!), Măceşului Valley (!), Latoriţa Valley (!), Mânăileasa Valley (!), Voineşiţa Valley (!), Voineasa (!);

Pulicaria dysenterica (L.) Bernh.: H, Ec; U4T3.5R0, Agropyro-Rumicion, Molinietalia; Brezoi (!);

Pulicaria vulgaris Gaertner: Th, Eua; U4T3R3, Agropyro-Rumicion, Bidention tripartiti, Isoeto-Nanojuncetea; Golotreni (30);

Senecio rivularis (Waldst. and Kit.) DC.: H, Ec; U4T2R2.5, Adenostylion alliariae, Filipendulo-Petasition, K: Sterpu Peak/Negru Hill (74); var. alatus (Jacq.) Cuf.: Sterpu Peak/Negru Hill (59, 90);

Telekia speciosa (Schreber) Baumg.: H, Carp-B-Cauc-Anat; U4T2R0, Alnion glutinosaeincanae, Filipendulo-Petasition, Telekion: Brezoi (30, !), Latoriţei Gorge (!), Ciungetu (!), Vidra Lake (!), Malaia (!), Păscoaia (!), Săliştea (!), Măceşului Valley (!), Latoriţei Valley (10), Mânăileasa Valley (!), Voineasa (!), Voineşiţa (!);

\section{Alismataceae}

Alisma plantago-aquatica L.: Hh, Cp; U6T0R0, Phragmitetea: Brădişor (!), Brezoi (!), Gura Lotrului (!), Săliştea (!), Mânăileasa Valley (!), Voineasa (!);

Potamogetonaceae

Potamogeton nodosus Poiret (P. fluitans Roth): Hh, Cp; U6T3.5R4, Potametalia; Gura Lotrului (!);

Orchidaceae

Dactylorhiza cordigera (Fries) Soo (Orchis cordigera Fries) ssp. cordigera: G, Carp-B; U4.5T2R2, Montio-Cardaminetalia, Scheuchzerio-Caricetalia nigrae; Gaura Mohorului (10), Setea Mare (41, 45), Lotrului Valley (45);

Dactylorhiza saccifera (Brongn.) Soo (D. maculata ssp. macrostachys (Tineo) Hayek): G, E; U4T2R2, Molinietalia: Vidra (27), Voineasa (27);

Epipactis palustris (L.) Crantz: G, Eua; U4.5T3R4.5, Caricetalia davallianae, Eriophorion latifolii, Molinion coeruleae: Obârşia Lotrului-Vidra (!), Voineasa-Măceşului Valley (!);

\section{Juncaceae}

Juncus articulatus L. (J. lampocarpus Ehrh.): H, Cp; U5T2R0, Agropyro-Rumicion, Calthion palustris, Nanocyperion flavescentis: Brădişor (!), Brezoi (!), Ciungetu (!), Groapa SeacăObârşia Lotrului (19), Vidra Lake (!), Obârşia Lotrului (12, 57, !), Măceşului Valley (!), Jidoaia Valley (!), Lotrului Valley, 510 m (7), Rânjeu Mare Valley (!), Voineşiţa Valley (!); Juncus bufonius L.: Th, Cosm; U4.5T0R3, Bidentetea tripartiti, Nanocyperetalia, Plantaginetalia majoris: Ciungetu (!), Vidra Lake (!), Obârşia Lotrului (!), Lotrului Valley, 510 m (7), Măceşului Valley (!), Mânăileasa Valley (!), Voineşiţa Valley (!), Vidra (!), Voineasa (!);

Juncus compressus Jacq.: G, Eua; U4T3R4, Agropyro-Rumicion, Agrostion stoloniferae, Nanocyperion flavescentis, Plantaginetea majoris, Puccinellio-Salicornietea: Brădişor (!), Brezoi (!), Gura Lotrului (!), Malaia (!), Păscoaia (!), Săliştea (!), Stan’s Valley (!), Măceşului Valley (!), Mânăileasa Valley (!), Rânjeu Mare Valley, 1,400 m alt. (!), Voineşiţa Valley (!), Voineasa (!); var. metzleri (F. Schultz) Ascherson and Graebner: Obârşia Lotrului (!), Vidra (!); 
Juncus conglomeratus L.: H, Cp; U4.5T3R3, Calthion palustris, Molinietalia, Molinion coeruleae, Scheuchzerio-Caricetalia nigrae: Brezoi (!), Curmătura Vidruței (!), Groapa Seacă-Obârşia Lotrului (19), Vidra Lake (!), Obârşia Lotrului (12, 57, !), Mânăileasa Valley (!), Voineasa (!);

Juncus effusus L.: H, Cosm; U4.5T3R3, Alnetea glutinosae, Bidentetea tripartiti, Calthion palustris, Molinietalia, Plantaginetea majoris: Brădişor (!), Brezoi (!), Ciungetu (!), Gura Lotrului (!), Vidra Lake (!), Malaia (!), Obârş̧ia Lotrului (57, !), Păscoaia (!), Săliştea (!), Lotrului Valley (1), Mânăileasa Valley (!), Rânjeu Mare Valley, 1,400 m alt. (!), Rânjeu Mic Valley (!), Voineşiţa Valley (!), Voineasa (!);

Juncus filiformis L.: H (G), Cp (arct-alp); U4.5T2.5R2.5, Caricion canescenti-nigrae; Câlcescu (2, 10), Iezeru, 1,935 m (2); var. transsilvanicus (Schur) A. and G.: Circul Câlcescu (14), Lotrului Valley (1);

Juncus inflexus L.: H, Eua; U4T3.5R4, Agropyro-Rumicion: Brezoi (!), Ciungetu (!), Gura Lotrului (!), Obârşia Lotrului (57), Măceşului Valley (!);

\section{Cyperaceae}

Blysmus compressus (L.) Panzer: G, Eua; U4.5T3R4.5, Agropyro-Rumicion, Caricion canescenti-nigrae, Lotrului Valley (1, 59), Mânăileasa Valley (!);

Carex acuta L. (C. gracilis Curtis) ssp. acuta: G (Hh), Cp; U5T3R0, Alno-Padion, Calthion palustris, Caricion gracilis, Magnocaricion elatae; Obârşia Lotrului (!);

Carex brunescens (Pers.) Poiret: H, Cp (arct-alp); U4T1.5R2, Cardamini-Montion, Scheuchzerio-Caricetea nigrae: Circul Câlcescu (14), Negru Hill (59, 73, 90, HF), Lotrului Valley $(1,59)$;

Carex curta Good. (C. canescens auct. non L.): H, Cp (bor); U5T0R2, Caricion canescentinigrae: Circul Câlcescu (2, 10, 14, 86), Negru Hill (59, 74, 90, HU), Groapa Seacă-Obârşia Lotrului (19, !), Iezeru (2, 10), Lunca cu Funiile (1, !), Muntinu Mare (2), Muntinu Mic (10, 86), Muşătoiu (2), Pravăţu Mare (1), Puru (10), Sărăcinu Mare (1), Sărăcinu Mic (1), Ştefanu (1), Urdele (2, 10), Lotrului Valley, 1,300 m (1, 59), Vidra (1, !), Zăvoi-Vidra (1, 1962), Zănoguța (2); f. tenuis O. F. Lang: Lotrului Valley (1, 59);

Carex distans L.: H, Eua (sAtl-sM); U4T3R4, Agrostion stoloniferae, Eriophorion latifolii, Molinion coeruleae: Brezoi (!), Obârşia Lotrului (!), Vidra (!);

Carex echinata Murray (C. stelllulata Good.): H, Cp; U5T2R1, Calthion palustris, Caricion canescenti-nigrae, Magnocaricion elatae: Circul Câlcescu (2, 10, 86), Groapa Seacă-Obârşia Lotrului (19, !), Iezeru (2, 10), Lunca cu Funiile (1, !), Mohoru (14), Muntinu Mic (86), Obârşia Lotrului (!), Pravăţu Mare (1), Sărăcinu Mare (1), Sărăcinu Mic (1), Ştefanu (2), Lotrului Valley, 130-1,400 m alt. (37, 59), Mânăileasa Valley (!), Ştefleşti Peak (68, !), Vidra (1, !), Voineasa (!), Zăvoi-Vidra (1); var. grypos Schk.: Lotrului Valley (1);

Carex elongata L.: H, Eua (bor); U5T2.5R4, Alnetea glutinosae: Lunca cu Funiile (1), Vidra (1), Lotrului Valley, 1,200-1,400 m alt. (1, 37, 59);

Carex flava L.: H, Cp; U4.5T3R0, Calthion palustris, Caricetalia davallianae, Eriophorion latifolii, Tofieldietalia: Câlcescu (2), Groapa Dengherului (12), Groapa Seacă-Obârşia Lotrului (19, !), Iezeru (2, 10), Muntinu Mic (2, 10), Muşătoiu (10), Obârşia Lotrului (!), Puru (10), Ştefanu (2), Lotrului Valley in Sphagnetum (1);

Carex lepidocarpa Tausch: H, Euram; U4.5T3R0, Eriophorion latifolii, Molinietalia, Montio-Cardaminetalia: Lotrului Valley (1);

Carex magellanica Lam. ssp. irrigua (Wahlenb.) Hiitonen (C. paupercula Michx.): G, Cp (arct-alp); U5T2R2, Scheuchzerio-Caricetea nigrae: Negru Hill (59, 74, 84, 90); 
Carex nigra (L.) Reichard (C. fusca All.) ssp. nigra: G, Cp; U4T3R2, Calthion palustris, Caricetalia davallianae, Caricion canescenti-nigrae: Groapa Dengherului (12), Obârşia Lotrului (!), Sărăcinu Mic (1), Negovanu Mare Peak (68, !); ssp. dacica (Heuffel.) Soo (C. dacica Heuffel, Carex bigelowii Torrey and Schwein.): G, Carp-B; U0T2R2,5; Caricion canescenti-nigrae: Circul Câlcescu (1, 2, 10, 14, 86), Negru Hill (90), Ierezu 1,935 m (2), Păpuşa (10); f. longifolia Circul Câlcescu (6, 14);

Carex ovalis Good. (C. leporina auct. non L.): H, Cp; U4T2.5R3, Caricion canescenti-nigrae, Molinietalia, Nardetalia: Brezoi (!), Cărbunele (10), Groapa Seacă-Obârşia Lotrului (19, !), Lunca cu Funiile (1, !), Vidra Lake (!), Malaia (!), Mohoru (2), Muntinu Mare (2), Muntinu Mic (10), Muşetoiu (2, 11), Obârşia Lotrului (57, !), Părăginosu (2, 86), Petrimanu (2), Pietrele (2), Pravăţu Mare (1), Sărăcinu Mare (1), Sărăcinu Mic (1), Ştefanu (2), Mânăileasa Valley (!), Urdele (2), Vidra (12), Zăvoi-Vidra (1), Zănoguţa (2);

Carex pallescens L.: H, Cp; U3.5T3R3, Molinio-Arrhenatheretea, Nardetalia: Groapa Seacă-Obârşia Lotrului (19), Muntinu Mic (10), Sărăcinu Mic (1), Urdele (10);

Carex panicea L.: G, Cp; U3.5T3R0, Magnocaricion elatae, Molinietalia, MolinioArrhenatheretea, Scheuchzerio-Caricetea nigrae, Tofieldietalia: Obârşia Lotrului (!), Voineşiţa Valley $(27,59)$;

Carex pauciflora Lightf.: G, Cp (bor); U5T2.5R1, Oxycocco-Sphagnetea, Sphagnion fusci: Câlcescu Lake (1, 8, 14), Lotrului Valley, 1,300 m alt. (37, 59);

Carex remota L.: H, Cp; U4.5T3R3, Alno-Padion, Fagetalia silvaticae: Brezoi (!), Golotreni (!), Săliştea (!);

Carex rostrata Stokes ssp. rostrata: H (Hh), Cp; U5T2R0, Caricion rostratae, Magnocaricion elatae: Câlcescu $(10,14)$, Obârşia Lotrului (!), Pravăţu Mare (Ştefureac et al., 1962), Ştefanu, 1,700-1,900 m (2, 10), Lotrului Valley (1, 59);

Carex vesicaria L.: Hh, Cp; U6T3R4, Caricion gracilis, Magnocaricion elatae: Câlcescu (14); Carex vulpina L.: H, Eua; U4T3R4, Agropyro-Rumicion, Caricion gracilis, Magnocaricion elatae, Phragmition australis; Brezoi (!), Golotreni (!), Gura Lotrului (!), Săliştea (!);

Cyperus flavescens Jacq. (Pycreus flavescens (L.) Reichenb.): Th, Cosm; U4.5T0R4, Nanocyperion flavescentis; Brezoi (27, 59, !);

Eleocharis palustris (L.) Roemer and Schultes: G (Hh), Cosm; U5T0R4, Molinietalia, Nanocyperetalia, Phragmitetea: Brădişor (!), Gura Lotrului (!), Săliştea (!), Mânăileasa (!);

Eriophorum angustifolium Honck.: G (Hh), Cp; U4.5T3R3, Molinietalia, ScheuchzerioCaricetalia nigrae; Lotrului Valley (37);

Eriophorum latifolium Hoppe: H, Cp; U5T0R4.5, Caricion davallianae, Eriophorion latifolii, Scheuchzerio-Caricetalia nigrae, Tofieldietalia; Circul Câlcescu (14), Groapa Dengherului (12), Obârşia Lotrului (!), Lotrului Valley (1);

Eriophorum scheuchzeri Hoppe: H, Cp (arct-alp); U5T1.5R2.5, Eriophorion scheuchzerii, Balindru Peak (HBZ), Negovanu Mare Peak (59, 74, 73, HF, HU);

Eriophorum vaginatum L.: H, Cp; U4.5T0R1.5, Sphagnion fusci: Circul Câlcescu (2, 10, 14, 86), Groapa Dengherului (12), Iezeru, 1,935 m (2, 10), Lunca cu Funiile (1, 6), Mohoru (14), Muntinu Mic, 1910 m (2), Obârşia Lotrului (!), Sărăcinu Mare (1), Sărăcinu Mic (1, !), Ştefanu, 1910 m (2), Negovanu Mare Peak (68, HBZ; HDRG, !), Ştefleşti Peak (68, !),

Schoenoplectus lacustris (L.) Palla (Scirpus lacustris L.): G (Hh), Cosm; U6T3R4, Phragmition australis; Gura Lotrului (!);

Scirpus sylvaticus L.: G, Cp; U4.5T3R0, Alno-Padion, Calthion palustris, Molinietalia, Phragmitetea: Brădişor (!), Brezoi (!), Ciungetu (!), Gura Lotrului (!), Malaia (!), Obârşia Lotrului (!), Săliştea (!), Măceşului Valley (!), Rânjeu Mare Valley (!), Voineşiţa Valley (!), Vidra (!), Voineasa (!), Lotrului Valley (1); 


\section{Poaceae}

Agrostis canina L. ssp. canina: H, Eua; U4T3R3, Caricion canescenti-nigrae, MolinioArrhenatheretea: Obârşia Lotrului (!), Lotrului Valley (1);

Agrostis stolonifera L. ssp. stolonifera: H, Cp; U4T0R0, Agropyro-Rumicion, Agrostion stoloniferae, Alno-Padion, Magnocaricion elatae, Molinion coeruleae: Brezoi (!), Ciungetu (!), Golotreni (!), Gura Lotrului (!), Malaia (!), Obârşia Lotrului (57), Stan’s Valley (!), Măceşului Valley (!), Lotrului Valley (1), Mânăileasa Valley (!), Voineasa (!);

Alopecurus aequalis Sobol.: Th-TH, Cp; U5T3R4, Agrostion stoloniferae, Bidentetalia tripartiti, Nanocyperion flavescentis: Gura Lotrului (!), Păscoaia (!), Săliştea (!), Mânăileasa Valley (!), Voineşiţa Valley (!), Voineasa (!);

Alopecurus geniculatus L.: Th-TH, Cosm; U5T0R4, Agropyro-Rumicion, Agrostion stoloniferae, Plantaginetea majoris: Brezoi (!);

Alopecurus pratensis L. ssp. pratensis: H, Eua; U4T3R0, Agrostion stoloniferae, Calthion palustris, Filipendulo-Petasition, Molinio-Arrhenatheretea: Malaia (!);

Calamagrostis pseudophragmites (Haller fil.) Koeler: H, Eua-C; U5T3R5, Salicion eleagni: Latoriţei Gorge (!), Ciungetu (!), Gura Lotrului (!), Malaia (!), Obârşia Lotrului (57, !), Vidra (!), Măceşului Valley (!), Mânăileasa Valley (!), Voineşiţa Valley (!), Voineasa (!);

Calamagrostis stricta (Timm) Koeler (C. neglecta auct non Ehrh.): H, Cp; U4.5T2R3, Magnocaricion, Caricion rostratae; Obârşia Lotrului-Vidra (1, !), Mânăileasa Valley (!);

Calamagrostis villosa (Chaix) J. F. Gmelin: H, Eua; U4T2.5R1.5, Calamagrostidion villosae, Vaccinio-Piceion; Urdele (10);

Deschampsia caespitosa (L.) Beauv. ssp. caespitosa (incl. ssp. alpicola Chrtek and Jirasek): H, Cosm; U4T0R0, Betulo-Adenostyletea, Molinietalia, Phragmitetalia: Ciungetu (!), Câlcescu (1, 2, 14), Coasta Bengăi 1,800 m (2), Curmătura Vidruței (!), Groapa Dengherului (12), Groapa Seacă to Obârşia Lotrului (19); Iezeru (1, 10), Vidra Lake (57, !), Miru Mare (1, 27), Mirăuţu Mountain (86, !), Mogoşu (86), Mohoru (2, 10, 11, 14), Muntinu Mare (2), Muntinu Mic (86), Muşătoiu (2, 10, 11), Nopteasa (2), Obârşia Lotrului (57, !), Păpuşa (71), Puru (2), Setea Mare (10, 11), Ştefanu (2), Urdele (2), Măceșului Valley (!), Haneşul Valley (!), Mânăileasa Valley (!), Pravăţu Valley (!), Voineşiţa Valley (!), Clăbucet Peak (68, !), Cristești Peak (!), Fratoşteanu Peak (!), Negovanu Mare Peak (68, !), Ştefleşti Peak (68, !), Voineagu Cătănesii (68, !), Voineasa (!), Zănoguţa (2);

Glyceria nemoralis (Uechtr.) Uechtr. and Koernicke: H, Ec; U5T3R3, Cardamini-Montion: Iezeru (10), Muşătoiu (10), Obârşia Lotrului (!), Mânăileasa (!), Voineasa (!), Voineşiţa (!); Glyceria notata Chevall. (G. plicata (Fries) Fries): H (Hh), Cp; U6T3R4.5, GlycerioSparganion: Groapa Seacă-Obârşia Lotrului (19), Păscoaia (!), Săliştea (!), Lotrului Valley (1), Măceşului Valley (!), Voineşiţa Valley (!), Voineasa (!);

Phragmites australis (Cav.) Steudel ssp. australis: G (Hh), Cosm; U6T0R4, Phragmition australis: Corbu (!), Gura Lotrului (!);

Poa palustris L.: H, Cp; U5T3R4, Alnetalia glutinosae, Calthion palustris, Magnocaricion elatae, Phragmition australis: Lotrului Valley (1), Mânăileasa Valley (!), Voineasa (!);

Typhaceae

Typha angustifolia L.: G (Hh), Cp; U6T4R0, Phragmition australis: Gura Lotrului (!);

Typha latifolia L.: G (Hh), Cosm; U6T3.5R0, Phragmition australis: Brădişor (!), Gura Lotrului (!), Stan’s Valley (!), Măceşului Valley (!), Mânăileasa Valley (!), Voineşiţa Valley, $700 \mathrm{~m}$ alt. (!). 


\section{CONCLUSIONS}

The aquatic and marshy flora of the Lotru River basin can be considered rich for a mountainous Carpathian area with its inventory of 204 taxa, and with the occurences of some rare species and species typical of the area. They are distributed particularly in smaller standing waters, bogs and springs and less represented in the water body of Lotru River. Due to the human impact caused by hydrotechnical constructions on the Lotru River and its tributaries, many of the natural habitats of the hydro- and hygrophilous flora have been entirely destroyed and have disappeared, others are still present in small area and frequently modified by human impact, but also some man-made new habitats have developed.

The present paper constitutes a documentary basis for the flora of the Lotru River basin, a useful species list for phytodiversity and comparative studies with other hydrographic basins.

REFERENCES (The numbers before the reference sources are codes used in the text for chorology of the taxa).

1. 24. Boruz V., 2004 - Speciile de Alchemilla L. conservate în herbarul Universităţii din Craiova (HUCv), Analele Universităţii din Craiova, Facultatea de Horticultură, (Volum omagial), VII (XLIII), 121-126. (in Romanian)

2. 57. Boruz V., 2006 - Chorology and ecology of the Alchemilla species in the Lotru Mountains, Acta Botanica Horti Bucurestiensis, 33, 85-92. (in Romanian)

3. 12. Boruz V., 2007 - Characterization of the species Alchemilla crinita, Cercetări ştiințifice, ser. a XI-a, Horticultură, inginerie genetică, Universitatea de Ştiințe Agricole şi Medicină Veterinară Timişoara, 342-351. (in Romanian)

4. 3. Boruz V., 2007 - Complex observations on the Alchemilla glabra species, Bulletin of University of Agriculture, Sciences and Veterinary Medicine Cluj-Napoca, Agriculture, 63, 171-176.

5. 5. Boruz V., 2008 - Speciile de Alchemilla L. din Carpaţii Meridionali cu referire specială la Masivul Parâng, Teză doctorat, Universitatea Bucureşti, Facultatea de Biologie, 309. (in Romanian)

6. 30. Brândză D., 1879-1883 - Prodromul Florei Române, Bucureşti, 566. (in Romanian)

7. 71. Buia A., 1943 - Contribuţiuni la studiul fitosociologic al păşunilor din Munţii Carpaţi, Buletinul Facultăţii de Agronomie, Cluj-Timişoara, X, Timişoara, 3-28. (in Romanian)

8. 2. Buia A., Păun M. and Pavel C., 1962 - Studiul geobotanic al pajiştilor în Pajiştile din masivul Parâng şi îmbunătăţirea lor, Institutul Agronomic „T. Vladimirescu” Craiova, Edit. Agro-Silvică Bucureşti, 143-274. (in Romanian)

9. Buia A., 1963 - Les associations a Nardus stricta L. de la R. P. R., Revue Roumaine de biologie serie de botanique, Bucureşti, VIII, 2, 113-137. (in French)

10. 10. Buia A., Păun M., Maloş C. and Olaru M., 1963 - Materiale pentru flora Masivului Parâng, Lucrările Grădinii Botanice Bucureşti, 1961-1962, 1, 267-297. (in Romanian)

11. 91. Ciocârlan V., 2000 - Flora ilustrată a României, Pteridophyta et Spermatophyta, Edit. Ceres, Bucureşti, 1139. (in Romanian)

12. 55. Ciurchea M., 1962 - Noutăţi floristice din raionul Râmnicu-Vâlcea, Studia Universitatis Babeș-Bolyai Cluj, seria Biologie, 1, 33-44. (in Romanian)

13. 44. Ciurchea M., 1963 - Flora teritoriului raionului Vâlcea din dreapta Oltului, Autoreferat, Univ. București, 31. (in Romanian)

14. Drăgulescu C., 1995a - The flora and vegetation of the Mureş (Maros) Valley, in Hamar J. and Sárkány-Kiss A. (edit), The Maros/Mureş River Valley, A study of the geography, hydrobiology and ecology of the river and its environment, Tiscia monograph series, Szolnok-Szeged-Târgu Mureş, ISBN 963-04-5103-4, 47-111.

15. 68. Drăgulescu C., 1995b - Flora şi vegetaţia din bazinul Văii Sadului, Edit. Constant Sibiu, 365. (in Romanian) 
16. Drăgulescu C. and Macalik K., 1997 - The aquatic and paludal flora and vegetation from the Criş/Körös Rivers' Valleys, in Sárkány-Kiss A. and Hamar J. (edit), The Criş/Körös Rivers' Valleys, A study of the geography, hydrobiology and ecology of the river and its environment, Tiscia monograph series, Szolnok-Szeged-Tîrgu Mureş, ISBN 963-04-9564-3, 47-80.

17. Drăgulescu C. and Macalik K., 1999 - The aquatic and paludal flora and vegetation from the Someş/Szamos Valleys, in Sárkány-Kiss A. and Hamar J. (edit), The Someş/Szamos River Valley, A study of the geography, hydrobiology and ecology of the river and its environment, Tiscia monograph series, Szolnok-Szeged-Tîrgu Mureş, ISSN 1418-0408, ISBN 963-037646-6, 77-104.

18. Drăgulescu C., 1999 - The hydrophilous and hygrophilous flora and vegetation from the upper and middle Olt River Valley, in Sîrbu I., Curtean A. and Bănăduc D. (edit), The upper and middle Olt River basin, Transylvanian Review of Systematical and Ecological Research, Sibiu, 1, 13-30.

19. Drăgulescu C., Fintha I., Mihály A. and Szabó A., 1999 - Wetland flora and vegetation of the Upper Tisa river valley, in Hamar J., Sárkány-Kiss A. (edit.), The Upper Tisa Valley, Preparatory proposal for Ramsar site designation and an ecological background Hungarian, Romanian, Slovakian and Ukrainian co-operation, Tiscia monograph series, Szeged, ISSN 1418-0408, ISBN 963-03-7645-8, 213-272.

20. Drăgulescu C., 2005 - The hydrophylous and hygrophilous flora and vegetation of Târnave rivers, in Curteran-Bănăduc A., Bănăduc D. and Sîrbu, I., 2005, The Târnava river basin, Transylvanian Review of Systematical and Ecological Research, 2, Sibiu, 13-30

21. 65. Fekete L. and Blattny T., 1913 - Az erdeszeti jelentosegu fak es cserjek elterjedese a Magyar allam teruleten, I-II, Selmecsbanya. (in Hungarian)

22. 72. Fuss M., 1853 - Bericht uber den Stand der Kenntnis der Phanerogamen Flora Siebenburgens mit dem Schlusse des Jahres 1853, Programm d. Gymnas. A. C. zu Hermannsradt f. d. Schuljahr 1851-1852, Sibiu, 1-31. (in German)

23. 73. Fuss M., 1866 - Flora Transsilvaniae Excursoria, Sibiu, 864.

24. 31. Grecescu D., 1898 - Conspectul Florei României, Bucureşti, 836. (in Romanian)

25. 27. Grecescu D, 1909 - Supliment la Conspectul Florei României, Bucureşti, 220. (in Romanian)

26. 13. Nyárády E. I., 1955 - Vegetaţia Muntelui Cozia şi câteva plante noi pentru flora Olteniei, Moldovei şi Transilvaniei, Buletin Știinţific secţia Știinţe biologice, agronomie, geologie și geografie, VII, 2, 219-246. (in Romanian)

27. 38. Oprea A., 2005 - Lista critică a plantelor vasculare din România, Edit. Univ. „Alexandru Ioan Cuza” Iaşi, 667. (in Romanian)

28. 32. Ploaie G. and Ştefureac T., 1983 - O viitoare rezervaţie botanică şi peisagistică: Piatra Târnovului (Judeţul Vâlcea), Ocrotirea Naturii și a Mediului Inconjurător, 27, 2, 135-138. (in Romanian)

29. 6. Ploaie G., 1983 - Valea Lotrului, Edit. Sport-Turism Bucureşti, 194. (in Romanian)

30. 25. Ploaie G., 1987 - Valea Latoriţei, Edit. Sport-Turism Bucureşti, 109. (in Romanian)

31. 8. Ploaie G., 1999 - Natura sălbatică, Edit. Prisma, Râmnicu Vâlcea, 129. (in Romanian)

32. 23. Ploaie G., Nicolescu E. and Ploaie G. Jr., 2004 - Date preliminare privind înfiinţarea unei rezervaţii naturale Dengheru-Cioara din Munţii Parâng, Analele Universităţii din Craiova, Facultatea de Horticultură (volum omagial), VII (XLIII), 111-120. (in Romanian)

33. 37. Pócs T., 1957 - Contributions à la flore des Carpathes Orientaux et Méridionaux, Annales Musei Historiae Naturalis Hungariae, VIII, Seria nova, 205-217. (in French)

34. 47. Pócs T., 1963 - Adatok a Déli-Kárpátok növénytakarójának ismeritéhez, Az Egri tanárképzö föiskola füzetei, 229-247. (in Hungarian) 
35. 4. Pócs T., 1961, 1962, 1967 - Flore du massif du Parâng (Carpathes méridionaux en Roumanie), Fragmenta Botanica Musei Historiae Naturalis Hungariae, 1, 49-128, 2, 73-130, 5, 69-98. (in French)

36. 19. Popescu G. and Boruz V., 2008 - Taxonomy, ecology and coenology data on the species Sisyrinchium montanum, Contribuţii Botanice Cluj-Napoca, XLIII, 39-44.

37. 92. Sanda V., Biţă D. and Barabaş C. N., 2003 - Flora cormofitelor spontane şi cultivate din România, Edit. „Ion Borcea” Bacău, 316. (in Romanian)

38. 59. Săvulescu T. (ed.), 1952-1976 - Flora Republicii Populare Române (Flora Republicii Socialiste România), I, II, III, IV, V, VI, VII, VIII, IX, X, XI, XII, XIII, Edit. Acad. Bucureşti, 660, 702, 662, 952, 556, 676, 662, 708, 1000, 752, 876, 810, 302. (in Romanian)

39. 90. Schur F., 1866 - Enumeratio plantarum Transsilvaniae, Vindobonae, 984.

40. 74. Simonkai L., 1886 - Enumeratio florae transsilvanicae vasculosae critica, Budapest.

41. 7. Ştefureac T., Popescu A. and Lungu L., 1955 - Contribuţii la cunoaşterea florei şi vegetaţiei briofitelor din Valea Lotrului, Buletin știinţific, Academia Republicii Populare Române, 7, 3, 525-588. (in Romanian)

42. 56. Ștefureac T., 1959 - Noi contribuţii la cunoaştera florei şi vegetaţiei bryophytelor din Valea Lotrului, Studii și cercetări de biologie, seria biologie vegetală, 11, 1, 7-61. (in Romanian)

43. 52. Ştefureac T., Lungu L. and Popescu A., 1959 - Specii de Sphagnum din câteva sfagnete de pe Valea Lotrului, Comunicările Academiei R. P. R., Bucureşti, IX, 1, 39-44. (in Romanian)

44. 1. Ştefureac T., Lungu L., Ţeculescu V. and Popescu A., 1962 - Specii de Carex şi Salix din sfagnetele de pe Valea Lotrului cu unele consideraţii geobotanice, Analele Universitătii Bucureşti, Seria ştiințe naturale-biologie, 33, 81-95. (in Romanian)

45. 14. Ştefureac T., 1969 - Studii briologice în unele formaţiuni de vegetaţie din România, Edit. Acad. Bucureşti, 163. (in Romanian)

Annex 1: Herbariums

BUCA Romanain Academy Biology Institute, Bucharest.

HBZ Herbarium J. Bielz, E. Krauss, G. Hergotta, V. Klotz - Brukenthal Museum Sibiu, Natural Sciences, no. inv. 79.911-81.464, 81.568-81.687.

HDRG Herbarium C. Drăgulescu Botanical Garden Cluj-Napoca.

HDRG Herbarium C. Drăgulescu Natural History Museum of Sibiu.

HF Herbarium M. Fuss (incl. Herb. Cryptogam. şi Herb. Norm. Transs.) - Brukenthal Museum of Sibiu, Şt. nat. no. inv. 1-28.983.

HFA Anmex Herbarium M. Fuss - Muzeul Brukenthal Sibiu, Şt. nat. no. inv. 82.435-84.427.

HK Herbarium E. Kisch - Muzeul Brukenthal Sibiu, Şt. nat. no. inv. 78.26-79.910.

HU Herbarium K. Ungar - Muzeul Brukenthal Sibiu, Şt. nat. no. inv. 35.520-43.288. 